new factor, called TR, is alleged to be altogether different. It is unlike G-factor in its physical behaviour, in that it has high thermal lability, and elutes at a different volume from an ion-exchange column (as assayed by activity). It does not require GTP. Ishitsura and Kaji conjecture that the new factor may be needed during chain termination. The termination factor, recognizing the final codon of the message, enters the acceptor site on the ribosome and releases the polypeptide chain, leaving the discharged tRNA in the donor site. The G-factor, or translocase, is then no longer of use, and it is the TR factor that releases the tRNA, so that the ribosomal subunits can dissociate in preparation for the next round of protein synthesis. It is not yet certain that the TR factor cannot be identified with one or other of the nine or so factors so far reported.

\section{ANIMAL VIRUSES}

\section{Cells Modified}

\section{from our Virology Correspondent}

The result of some very rapid research on RNA tumour viruses by Professor S. Spiegelman (Institute of Cancer Research, New York) was the last but most exciting report at the Royal Society meeting on animal viruses as genetic modifiers of the cell, held on June 18. After a general introduction on the present hypotheses of mechanisms of infection by RNA tumour viruses, he went on to report his work performed since the international cancer congress at Houston (May 22-29), at which Dr H. M. Temin suggested that RNA can act as a template for DNA synthesis (Nature,226, 1003, and page 1211 in this issue). Spiegelman showed this to be true by demonstrating that the product obtained from the action of polymerases in disrupted preparations of various tumour viruses (including avian myeloblastosis virus and feline leukaemia virus) was in fact DNA. This was shown by its resistance to RNase and pronase, sensitivity to DNase and by buoyant density determination.

As a consequence of this, Spiegelman proposed a mechanism to explain the occurrence of leukaemia in mice (known to be transmitted through sperm and egg), in which mouse genome carries sequences of DNA complementary to the viral RNA and which are repressed in the normal state. If derepression occurs (for example, due to ageing) then the virus is produced and leukaemia develops. That these tumour viruses exhibit a reaction which could be specifically inhibited opens up interesting possibilities in cancer therapy.

The rest of the meeting covered a fairly broad field and opened with Professor D. C. Burke (University of Warwick) discussing the present state of knowledge regarding interferon production by animal cells. The mechanism of stimulation of interferon by virus is unknown, but the most likely hypothesis is that the viral nucleic acid acts on a membrane site which triggers a signal to the nucleus, rather than the virus actually triggering the nucleus itself.

The antigenic changes which occur when a cell is transformed by a virus were the subject of several reports. Of particular interest was the work of $\mathrm{Dr}$ A. C. Allison (National Institute for Medical Research, London), who showed that there was greater tumour development from polyoma virus infection in immuno- suppressed mice than in normal animals, but that it was possible to overcome this by restoration with sensitized lymphoid cells. The possible relevance of cell-mediated immunity to man was illustrated by the finding that in renal homograft patients who were immunosuppressed, there was a significantly higher incidence of tumours than in normal individuals, although it was not known if they were virus induced.

Professor P. Berg (Stanford University) assessed the evidence for the integration of polyoma viral DNA into host cell genome and cited $\lambda$ phage lysogeny as a suitable model (although he did note that in $\mathrm{P}_{1}-E$. coli lysogeny no integration occurs). He showed that there was a greater transforming efficiency by oligomeric forms of polyoma DNA and suggested mechanisms for formation of these. The use of temperature sensitive mutants as an important tool for investigating mechanisms in this field was shown by Berg and other speakers.

\section{BRAIN}

\section{Memory and Disorders}

\section{from a Correspondent}

INDICATIONS of a biological difference between normal and schizophrenic subjects have come from experiments carried out at Galesburg State Research Hospital. At a symposium on the biochemistry of brain and memory, held at the University of Wisconsin, Parkside, on May 25 and 26, Dr H. E. Himwich described how he and his colleagues correlated behavioural and biochemical changes in chronic schizophrenic patients receiving L-cysteine, a sulphur amino-acid, and tranylcypromine ('Parnate'), a monoamine oxidase inhibitor. They found a rough correlation between the intensity of the schizophrenic symptoms and the urinary concentrations of three potentially psychotogenic $\mathrm{N}$ dimethyltryptamines. In the same experimental conditions, normal subjects, however, did not eliminate these urinary products nor did they have any schizophrenic-like symptoms.

Dr H. A. Waisman (University of Wisconsin Medical School) said that knowledge of mental retardation has advanced further than that of schizophrenia, for in some cases mental retardation has been definitely linked with genetic disorders and biochemical defects. Phenylketonurea, he said, was such an example, and one which can be remedied. He pointed out, however, that even for this disorder we do not know the mechanism by which excessive phenylalanine evokes its pathological effects. Dr A. J. Mandell (University of California, San Diego), discussing drugs which affect brain excitability, presented evidence that new enzymes are probably synthesized in cell bodies and transported to the nerve endings where they may help to regulate the rate of neurotransmitter biosynthesis.

The session on the biochemistry of memory was concerned with the formation of long term memory, for apparently little is known about biochemical action with respect to recent recall. Dr H. Hyden (University of Gothenberg) has studied the variations in the concentration of an acidic S100 protein, specific for the brain. When rats were trained to some new behaviour, there was an increase of the protein in the hippocampus after 8 and 14 days, which had returned to control values after 30 days. He suggested that the storage 